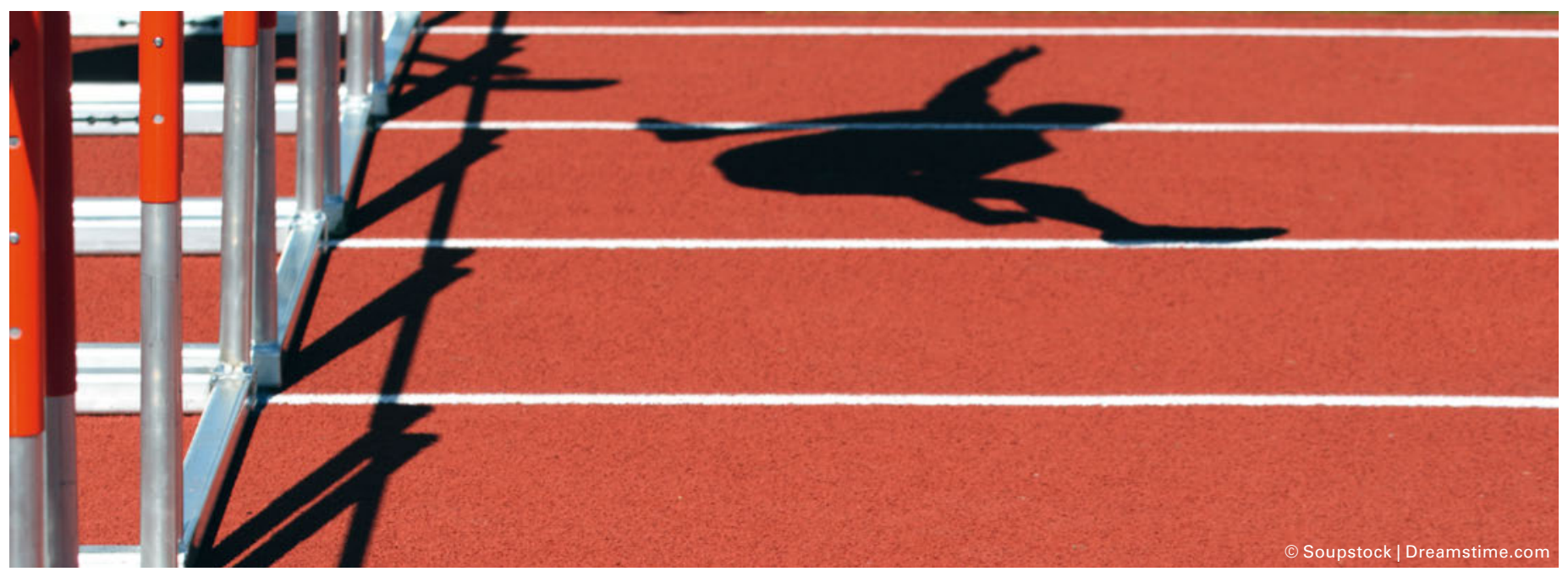

\title{
«Vom Sprung über den eigenen Schatten und andere Hindernisse»
}

\author{
Marco Zalunardoa, Sebastian Krayer ${ }^{b}$, Bernhard Walder ${ }^{c}$, Thomas Brunnerc, Peter Bauerfeind ${ }^{d}$, \\ Stefan Hartmeierd, Daniel Franzene, Ludwig Jacobf ${ }^{f}$ Peter Ammanng, Daniel Weilenmann ${ }^{g}$ \\ a Präsident SGAR; ${ }^{b}$ Vorstandsmitglied SGAR; ${ }^{\circ}$ Delegierter der SGAR; ${ }^{d}$ Vorstandsmitglied der SGG; ${ }^{e}$ Delegierter SGP; ${ }^{f}$ Vorstandsmitglied der SSVIR; \\ g Delegierter der SGK
}

2011 hatten Gastroenterologen im Swiss Medical Forum einen optimistischen Artikel mit dem Titel «Propofolsedation in der gastrointestinalen Endoskopie - eine Schweizer Erfolgsgeschichte» publiziert [1]. Das Thema, das den Praxisalltag in der Gastroenterologie widerspiegelte, hatte schon zuvor auf europäischer Ebene eine heftige Debatte ausgelöst, die damit endete, dass manche Anästhesisten und ihre nationalen Gesellschaften den Gastroenterologen die Kompetenz für den Umgang mit Propofol ganz absprachen [2]. Auf europäischer Ebene konnte kein Konsens erreicht werden. Eine entsprechende Reaktion in der Schweiz liess nicht lange auf sich warten. Einige Anästhesisten reagierten in einem Leserbrief auf den obgenannten Artikel in ähnlicher Weise gegenüber den Gastroenterologen [3]. Somit war der standespolitische Kleinkrieg lanciert und die Positionen waren bezogen. Eine grundsätzliche Kooperation von Anästhesie und Gastroenterologie in diesem Bereich erschien zu dieser Zeit an vielen Orten schwierig, auf standespolitischer Ebene gar unmöglich.

Der Initiative und der Überzeugungskraft einzelner Vorstandsmitglieder der beiden Fachgesellschaften war es zu verdanken, dass sich die Fachvorstände der
SGAR und der SGG trotz widriger Umstände zur Aussprache zu diesem Thema zusammengesetzt haben. Im August 2012 hat man sich zum ersten Mal getroffen. Die Atmosphäre war angespannt, beide Seiten rechneten mit Widerstand oder gar Eskalation. Nichts dergleichen trat ein. Eine offene Diskussion mit Bekenntnissen auf beiden Seiten: die Voraussetzung für eine fruchtbare Zusammenarbeit. Man war offenbar bereit über den eigenen Schatten zu springen. Dies sollte der Startschuss zu einer anhaltenden erfolgreichen Kooperation werden.

\section{Das primäre Ziel der gebildeten Arbeitsgruppe} aus der SGG und der SGAR war die Patientensicherheit.

Das primäre Ziel der gebildeten Arbeitsgruppe aus der SGG und der SGAR war die Patientensicherheit. In mehreren Sitzungen konnte schliesslich ein Konsensuspapier mit Empfehlungen und Richtlinien zur Sedierung mit Propofol durch die Gastroenterologen erstellt werden. Das Dokument wurde als gastroenterologische Richtlinie 2013 an der Generalversammlung der SGG trotz Widerstand genehmigt und übernom- 
men. Letztlich waren Verantwortlichkeit, juristische Konsequenzen und das Wohl und die Sicherheit des Patienten ausschlaggebend für die Annahme durch die anwesenden Mitglieder der SGG.

Der Gedanke lag nahe, sich nicht nur auf die Gastroenterologie und Propofol zu beschränken, sondern allgemein gültige Richtlinien und Safety Standards zu definieren, die unabhängig vom Interventionalisten und von der verwendeten Substanz anwendbar waren. So wurde die Arbeitsgruppe erweitert, und zwar einerseits mit spezialisierten Anästhesisten, die eine grosse Erfahrung bei Analgosedierungen für Interventionen mitbrachten und andererseits mit weiteren Fachgesellschaften, die regelmässig und häufig Analgosedierungen durchführen: Kardiologie, Interventionelle Radiologie und Pneumologie. Die erweiterte Arbeitsgruppe startete im Oktober 2014 unter der Schirmherrschaft der SGAR. Von allen beteiligten Fachgesellschaften wurden offizielle Delegierte geschickt. In dieser Runde herrschte seit Anbeginn ein konstruktives Klima; vielleicht auch, weil einige Delegierte bereits auf jahrelange erfolgreiche multidisziplinäre Zusammenarbeit zurückblicken konnten. Wichtige Diskussionsthemen beinhalteten zum Beispiel die vorausgehende Beurteilung der $\mathrm{Pa}$ tienten und deren Risiken, die «erlaubte» Sedierungstiefe, sowie die Nüchternzeit bis zum Eingriff/Sedierung. Eine moderate Sedierung (Stufe I und II) stellt aus anästhesiologischer Sicht noch keine besonderen Anforderungen an den Interventionalisten. Bei einer

\section{Der Gedanke lag nahe, sich nicht nur auf die Gastroenterologie und Propofol zu beschränken, sondern allgemein gültige Richtlinien und Safety Standards zu definieren.}

tiefen Sedierung (Stufe III und IV) verliert der Patient zunehmend das Bewusstsein. Die damit einhergehende Beeinträchtigung der Spontanatmung, der Schutzreflexe und Kreislaufstabilität erfordern gewisse Fähigkeiten im Erkennen und Behandeln einer unzureichenden Atmung und Kreislaufsituation. In der klinischen Praxis wird etwa das Einführen eines Gastroskopes im weckbaren Zustand (Sedationsstufen I und II) von den Patienten schlecht toleriert und auch kaum mehr akzeptiert. Die Ansprüche der Patienten sind in den letzten Jahren stark gestiegen, weshalb eine temporäre tiefere Sedierung für viele, insbesondere auch kardiologische Interventionen nicht nur wünschenswert ist, sondern seit Jahren auch einen festen Bestandteil der täglichen Praxis darstellt.
Diese Diskussion wurde in der Arbeitsgruppe intensiv geführt. Das Resultat wurde als Konsensuspapier wiedergegeben und schliesslich von allen Fachgesellschaften akzeptiert, auch von der Generalversammlung der SGAR, wobei Gegenwind zu spüren war. Im unmittelbaren Vorfeld und an der Generalversammlung gab es Vorstösse und Aktivitäten in einer Art und Weise, wie sie die SGAR noch nie erlebt hatte. Im Vordergrund standen vor allem die Angst vor Verlust von Einfluss, Kontrolle, Sicherheit, Qualität und natürlich auch Einnahmequellen. Daneben waren Verantwortlichkeit und allfällige juristische Konsequenzen ein Thema. In einer langen Diskussion konnte jedoch eine Mehrheit der anwesenden Mitglieder überzeugt werden, die Zeichen der Zeit zu erkennen und diese multidisziplinäre Zusammenarbeit zu unterstützen.

\section{Das Gelingen hängt in nicht zu unterschätzen-} dem Masse von den beteiligten Einzelpersonen ab.

Dieses Dokument, welches im Swiss Medical Forum parallel zu diesem Artikel publiziert wird, ist der erste gemeinsame Schritt [4]. Revisionen und Anpassungen werden nötig sein, um den sich verändernden klinischen Bedürfnissen gerecht zu bleiben. Der nächste Schritt ist die praktische Umsetzung in den einzelnen Fachgesellschaften. Hier wird die SGAR die anderen Disziplinen aktiv unterstützen und begleiten im Hinblick auf eine sinnvolle und nachhaltige Instruktion und Fortbildung ihrer Mitglieder auf der Basis des Konsensuspapiers.

Diese «Erfolgsgeschichte» zeigt einmal mehr, dass scheinbar unüberwindbare Hürden mit Überzeugung, Hartnäckigkeit und vor allem Konsensbereitschaft übersprungen werden können. Das Gelingen hängt in nicht zu unterschätzendem Masse von den beteiligten Einzelpersonen ab. Steht nicht die Sache, oder genauer der Patient, im Vordergrund, sondern Eigeninteresse und Profilierung, dann wird eine Konsensfindung unwahrscheinlich.

\section{Literatur}

1 Inauen W, Külling D, Heuss LT, Bauerfeind P. Propofolsedation in der gastrointestinalen Endoskopie - eine Schweizer Erfolgsgeschichte. Schweiz Med Forum. 2011;11(51-52):961-2.

2 Perel A. Non-anaesthesiologists should not be allowed to administer propofol for procedural sedation: a Consensus Statement of 21 European National Societies of Anaesthesia. Eur J Anaesthesiol. 2011;28(8): 580-4.

3 Knüsel R, Meister B, Schnider T. Propofolsedation in der gastrointestinalen Endoskopie: eine gefährliche Erfolgsgeschichte. Schweiz Med Forum 2012;12(25):519-21.

4 Zalunardo M, Krayer S, Brunner T, Walder B, Bauerfeind P, Hartmeier S, Ammann P, Weilenmann D, Jacob AL, Franzen D. Konsensuspapier der SGAR, SGG, SGK, SSVIR und SGP: Empfehlungen und Standards für die Analgosedierung durch Nicht-Anästhesisten. Schweiz Med Forum. 2016;16(45):969-72. 